\title{
CEREBRAL ANGIOGRAPHY IN NEUROSURGICAL EMERGENCIES
}

\author{
R. Myles Gibson, M.D., M.Sc., F.R.C.S. \\ Senior Registrar, Department of Neurological Surgery, The General Infirmary at Leeds \\ Michael D. Sumerling, B.Sc., M.B., Ch.B., M.R.C.P., D.M.R.D., F.F.R. \\ Research Fellow in Radiodiagnosis, University of Leeds
}

Cerebral angiography has established itself as a valuable diagnostic method in neurosurgical clinics. Since the introduction of this procedure by Moniz in 1927 the technique and the contrast media used have been modified and improved. Consequently the standard and diagnostic value of the radiograms obtained have been improved with, simultaneously, a reduction in the risks involved to the patient.

Angiography has played an important role in the surgical management of spontaneous subarachnoid haemorrhage and is now accepted as the routine method of investigation of such cases. The role of surgery in a case of spontaneous intracranial haemorrhage is to save the patient's life, to promote as full a recovery of function as possible and to prevent a further haemorrhage. Surgical intervention cannot succeed in the case of a mass lesion which kills the patient within 24 hours. However, in those that survive, surgical evacuation of haematomas may avert death by cerebral compression, diminish the severity of the hemiparesis and speed the rate of recovery. Often the aneurysm can be successfully obliterated, thus preventing another haemorrhage. Angiography is essential in ordèr to demonstrate the aneurysm or angioma, revealing its vessel of origin, its precise situation and size. Angiography may also demonstrate intracranial clots, the degree and extent of vasospasm, and furthermore may be used to show to what extent the circulation in one hemisphere can be maintained throughout the circle of Willis, when ligation of the parent carotid artery is the treatment of choice.

Carotid angiography is a valuable investigation of cases of cerebral neoplasms where the clinical signs indicate the cerebral hemisphere involved. Angiography in such cases is generally acknowledged to be less disturbing to the patient than ventriculography. Occasionally, too, it may provide useful information about the particular type and size of the cerebral tumour, depending on the vascular pattern of the lesion, and so facilitate a definitive surgical approach.

While the part played by carotid angiography in the above condition is well recognized and accepted, it is further submitted that angiography carried out under the proper conditions of clinical assessment has a valuable part to play in the elucidation of some 'emergency' clinical problems of altered consciousness.

There are, of course, many causes of coma or impaired consciousness. Such cases upon admission to hospital must be subjected to careful clinical scrutiny and the general causes of impaired consciousness such as diabetes, hypoglycaemic coma, uraemia, hepatic failure, alcoholism, poisoning, meningitis, recent head injury, eliminated. The three main requirements in unravelling the cause of the coma are an account from relatives or a witness of the patient's conduct prior to the loss of consciousness, a description of the mode of onset, and a thorough physical examination, including blood-pressure recordings, urinalysis and blood examination. This overall concept of the investigation of altered consciousness must be borne in mind to avoid errors in diagnosis and treatment. None the less, a busy general hospital admits a significant number of patients suffering acute or subacute alteration of consciousness, or hemiplegia, due primarily to disorders of the nervous system. These include spontaneous intracranial haemorrhage due either to rupture of an aneurysm or angioma, already mentioned, or to so-called primary cerebral haemorrhage with intracerebral clot, occlusion of the carotid artery in the neck or in the intracranial portion, subdural haematoma, brain tumour and intracranial abscess.

Much can be done to help patients, admitted to hospital in coma or semi-coma, who are suffering from these conditions. In the past, neurosurgical 
aid has depended primarily on accurate clinical assessment supplemented by multiple exploratory burr holes, and, if indicated, needling of the brain with a brain cannula. Occasionally ventriculography would be performed to locate or define a space-occupying lesion. Without detracting from the value of exploratory burr holes this is a blind procedure often involving multiple incisions over the cranial vault, and is frequently unrewarding. Furthermore, ventriculography in the very ill patient may well precipitate disaster. Angiography, on the other hand, may contribute a more precise and localized diagnosis and permit a planned, logical and definitive surgical approach to evacuate intracranial space-occupying lesions, and to relieve intracranial pressure or vascular occlusion. It is possible that in the near future the use of pulsed ultrasound may detect mass space-occupying intracranial lesions rapidly and without hazard, but the value of this method is as yet undetermined, and in any event it yields no information as to the source of the lesion or site of a vascular occlusion.

Carotid angiography, especially in the ill patient, is not without risks, but these are minimal under proper conditions of case assessment and cooperation between neuroradiologist and neurosurgeon, and the benefit to the patient far outweighs the risk. The hazards of angiography have recently been well reviewed by Riishede $^{3}$ and are worth briefly summarizing.

I. Deleterious effects of faulty or indiscriminate needle puncture-arterial spasm, intimal dissection and haematoma.

2. Injection of an unnecessarily large volume of radio-opaque medium may have a direct action on the cerebral tissues and may produce cerebral oedema or infarction of brain tissue. An unnecessarily large volume of dextrose solution injected to maintain needle patency while waiting between injections of media is also harmful, as well as bad practice, and can be eliminated by planned team work which enables speedy and safe angiography to be carried out.

3. The medium may have general toxic or allergic effects on the cardiovascular, excretory or other systems.

Most of the complications have followed the use of Diodone in relatively large amounts and often under general anaesthesia. These factors are of considerable importance in space-occupying lesions where cerebral oedema and mass shift of intracranial structures can take place rapidly, sometimes with serious and irreversible consequences. Fortunately, considerable advances have been made in the development of radiographic media in recent years. The modern media (notably Urografin, Diagonal and Hypaque) are less toxic and irritant. ${ }^{1}$ Moreover, they contain more iodine per molecule and so give better contrast per unit volume or conversely permit adequate visualization on a smaller total dosage injected.

\section{Technique}

Carotid angiography may be required at any time of day or night, and it is therefore necessary to have a radiological team available for emergency calls. An emergency sterile drum containing the necessary equipment is maintained, and this should include Gentile needles of various sizes, polyvinyl connections, steel bowls, syringes, gauze, swabs and sheets. Solutions of $2 \%$ Xylocaine, ampoules of $60 \%$ Urografin and bottles of $5 \%$ Dextrose perfusing fluid are always available.

In most patients sedation is necessary; we prefer Omnopon $1 / 6-1 / 3$ gr. and Scopolamine I/300-I/I 50 gr. rather than Pethidine or Chlorpromazine as the latter drugs are less predictable in action, and are more likely to produce a fall in the systemic blood pressure. A clear airway must be maintained and suction apparatus must be available since patients may vomit, particularly those with raised intracranial pressure.

The tissues are infiltrated with $2 \%$ Xylocaine and the carotid artery is punctured with a No. 18 Gentile needle attached to a polyvinyl connection filled with perfusing fluid. A clear puncture is particularly desirable as these patients are often critically ill. Modern media have greater contrast and less irritant action, and the injection of 4-6 ml. of $60 \%$ Urografin produces angiograms of adequate quality. Two serial antero-posterior films are first exposed to determine any shift of the anterior cerebral artery. This is followed by a second injection of medium with the exposure of three serial films. Correct positioning of the head is essential. Some movement of the head may take place, but this almost invariably happens at the end of the injection, and the first film shows good diagnostic detail.

Helpful information is usually furnished by an angiogram of one side, but on occasions it has been necessary to perform bilateral carotid angiography. In some patients where the provisional diagnosis is uncertain before angiography, or where extracranial carotid occlusion is suspected, it is important to insert the needle into the common carotid artery low in the neck. Films showing the carotid bifurcation may then be obtained, and in both antero-posterior and lateral views if necessary.

Blood pressure should be recorded before and after injections of media. Falls of pressure occur particularly where an aneurysm is present, and such falls if severe may precipitate infarction. ${ }^{2}$ 


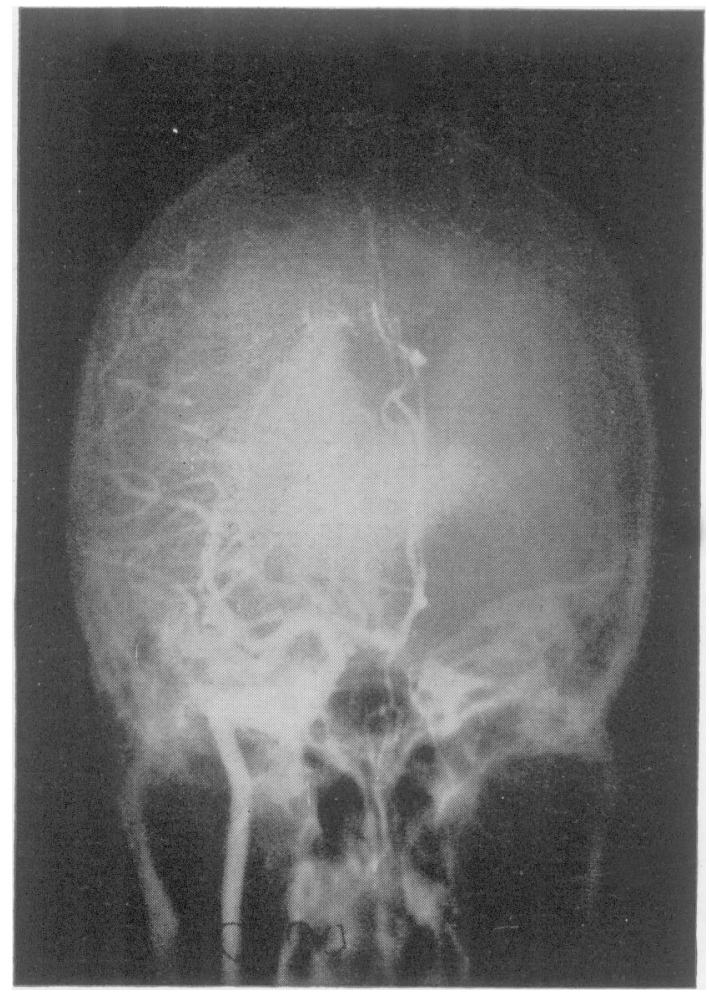

FIG. 1.-Right carotid angiogram. Antero-posterior film. The anterior cerebral artery is shifted to the left.

The whole procedure should be done as quickly as possible, consistent with safety, and the importance of good team work cannot be overstressed. Co-operation between the neurosurgeon and the radiologist in the planning of the procedure and the immediate interpretation of the films is essential, and enables the theatre staff to be alerted at an early stage. The patient may then be transferred directly from the X-ray department to the operating theatre without delay.

\section{Case No. I}

A housewife, aged 38 years, who enjoyed good health until the evening of May 5, I958, when she developed a sudden violent headache in the right temporal region. She went to bed; half an hour later experienced paraesthesiae in the left arm and after a further half-hour became unconscious. During the course of the night she vomited several times. The following morning she was admitted to the General Infirmary at Leeds. Lumbar puncture was performed, and the C.S.F. was clear and the cells reported as 7 lymphocytes, 50 red cells. Shortly after the lumbar puncture she developed stertorous breath-

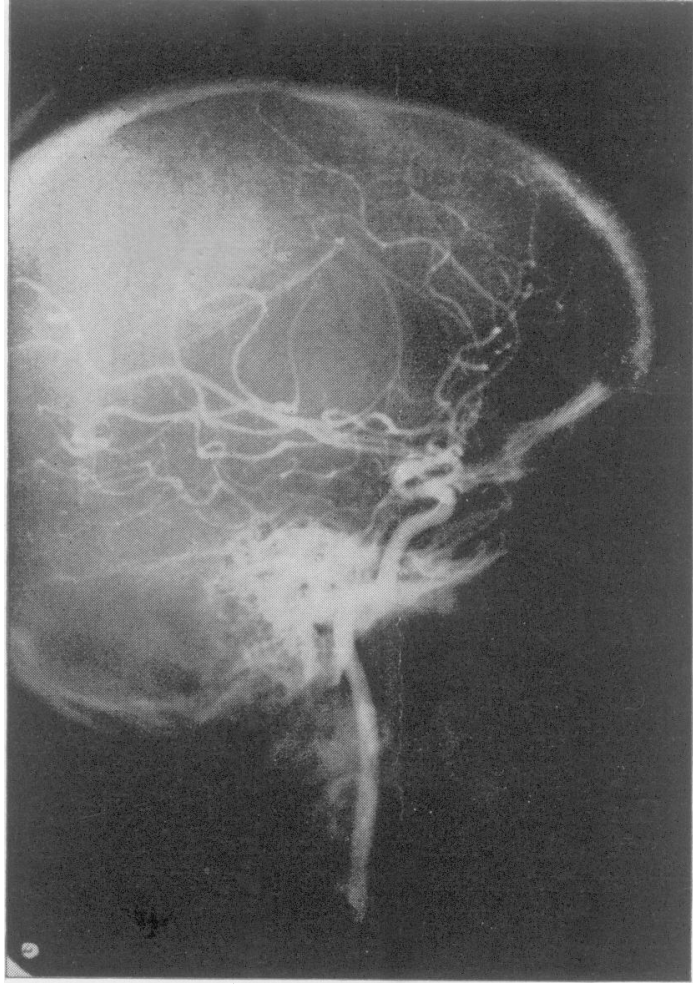

FIG. 2.-The lateral film shows stretched vessels around an avascular space occupying lesion in the parieto-temporal area.

ing and had several generalized seizures. She was then referred to the Department of Neurological Surgery. On examination at 7 p.m. on May 6, I958, she was very ill with stertorous breathing. The right pupil was larger than the left and in the mid-dilated position. She responded only to painful stimuli and made attempts to ward off pin-pricks with the right limbs but not with her left arm, and only infrequently with her left leg. Both plantar responses were extensor.

Interrogation of the patient's relatives confirmed the sudden onset of the patient's illness and clinical examination, having excluded general factors, strongly suggested a cerebral catastrophe involving the right cerebral hemisphere.

Right carotid angiography was performed under local anaesthetic. This showed a gross shift of the anterior cerebral artery from right to left (Fig. I), and in the lateral view (Fig. 2) the middle cerebral artery was displaced downwards. The pattern of vascular displacement, and the stretching of the vessels, suggested a moderate-sized right parieto-temporal avascular space-occupying lesion. 


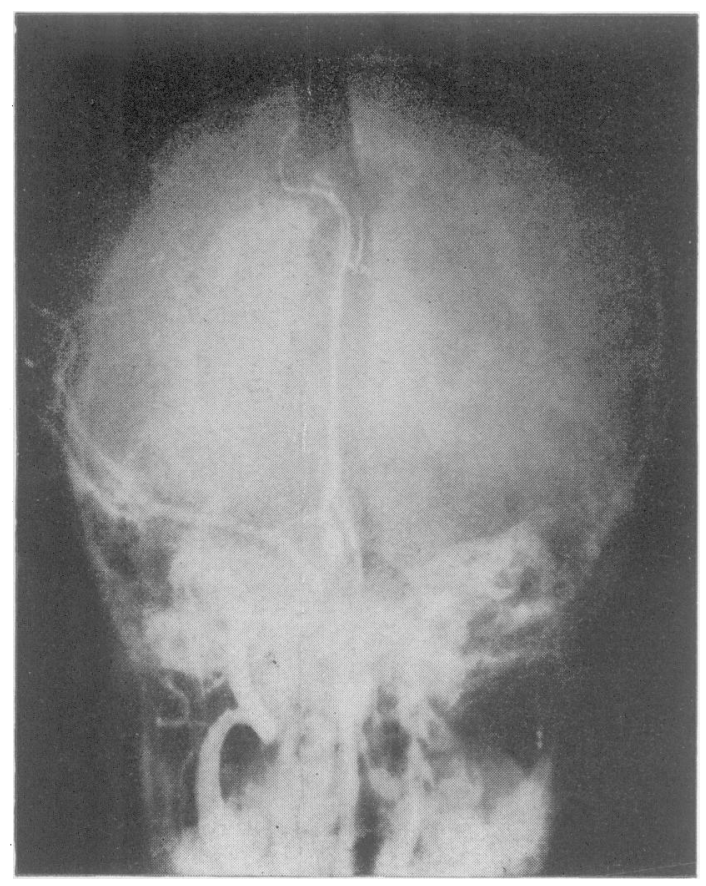

\section{Operation}

She was taken direct from the Department of Radiodiagnosis to the operating theatre. An endotracheal tube was passed and a tracheobronchial toilet effected. This improved the airway and she was given oxygen and a light anaesthetic. An extensive right lateral craniotomy was performed and a bone flap turned. The dura was slightly blue and very tense. It would have been dangerous to attempt opening the dura widely at this stage, and so a brain cannula was passed into the parietal region of the brain. At the depth of $1.5 \mathrm{~cm}$. fluid blood was encountered and easily aspirated. On account of its volume accurate measurement was impossible, but it was estimated to be about 80-100 c.c. The dura was then widely opened as a flap hinged towards the mid-line of the skull. The brain was now seen to be slack and pulsatile. Having thus evacuated the space-occupying lesion and afforded the patient a decompression against the possibility of brain swelling, the bone flap was laid gently in place and the wound closed in layers. At the completion of the operation tracheotomy was performed. The patient was nursed through a period of coma lasting ten days. She then slowly emerged from her coma, and was discharged from hospital two months later. As might be expected from the findings at the operation she had a moderately. severe left hemiparesis for which out-patient physiotherapy and rehabilitation were arranged.

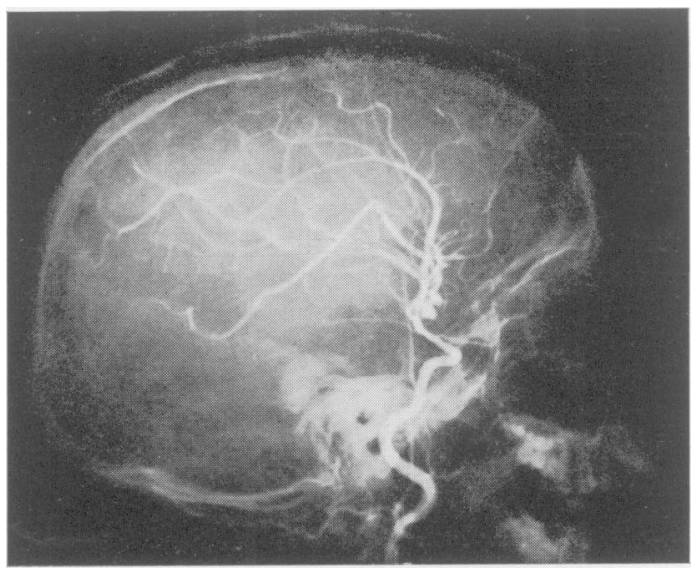

FIGS. 3 and 4.-Right carotid angiogram. The anterior cerebral artery is in the mid-line. A berry aneurysm has been demonstrated on the anterior communicating artery. There is considerable stretching of the arterial tree due to hydrocephalus. There is outward displacement of the bone flap, seen more easily on the lateral view.

Followed up regularly at out-patients this lady has made an excellent recovery. She is able to walk with the aid of only a stick, and despite the weakness of the left arm she is capable of looking after her own house and family.

Case No. 2

An II-year-old schoolboy, admitted to the General Infirmary at Leeds on February 23, 1958. He had two attacks of spontaneous intracranial haemorrhage, the first on February $I_{3}$ and the second the day prior to admission, February 22. On examination he was a sick, restless child; there was evidence of a left hemiparesis. Examination of the fundi disclosed early papilloedema. At this point it was felt unsafe to perform angiography and multiple burr holes were made. In the right parietal region an intracerebral haematoma of I4 c.c. was encountered at a depth of $4.5 \mathrm{~cm}$. and evacuated. Initially this helped the patient, but within 24 hours he was displaying signs of a marked rise of intracranial pressure. $\mathrm{He}$ was no longer responsive to pin-prick and there was a marked increase of tone in the legs associated with bilateral extensor plantar responses. Under a light anaesthesia, in which a high concentration of oxygen was maintained, an extensive right lateral bone flap was turned, the dura opened and the swollen brain decompressed. At the same time tracheotomy was performed, since it was anticipated that he would be in coma or semi-coma for some time. Slowly he improved, becoming responsive to painful stimuli and then making spontaneous movements of his limbs. 
This progress became interrupted about two and a half weeks later when it was noticed that the decompression was bulging and the bone flap beginning to ride.

This patient had had two proven attacks of subarachnoid haemorrhage complicated by an intracerebral haematoma and swollen brain. Surgery in the first irstance was directed towards saving the boy's life by removing the intracerebral clot and providing a satisfactory cerebral decompression. That all was not well after two and a half weeks was evident by the bulging decompression. The possibilities were those of renewed intracranial bleeding or haematoma collection, brain swelling, or hydrocephalus due to difficulty in C.S.F. disposal. Since the method of surgical treatment differed in each case it became of vital importance to clarify the situation. Right carotid angiography was performed under local anaesthesia. This showed a classical picture of hydrocephalus. The anterior cerebral artery was swept high but there was no evidence of intracerebral haematoma. Displayed also was a moderate-sized anterior communicating aneurysm, the probable source of the spontaneous haemorrhage (Figs. 3 and 4). Based on the information furnished by the angiography, a left frontal burr hole was made and continuous ventricular drainage instituted for six days. Lumbar puncture was also carried out daily. By these measures large quantities of blood-stained cerebrospinal fluid were removed and quickly the decompression ceased bulging and the bone flap settled into proper position. There was no need to reopen the craniotomy.

Over many months the boy slowly improved and was finally discharged home. Mentally he was fully alert and fit to resume his schooling. There was weakness of the legs, however, which for the present and immediate future requires him to move from place to place in a wheelchair, but his re-education and rehabilitation are being vigorously pursued.

\section{Case No. 3}

A 75-year-old lady was admitted to the General Infirmary at Leeds on August 2 I, 1958. She had sustained a mild head injury ten days before and two days later was admitted confused and disorientated. She had become apathetic and finally stuporous, and had in addition several focal fits involving the left limbs.

The patient's general health was fair, urinalysis revealed no abnormality, and the blood urea estimation was within normal limits. The history of mild head injury ten days previously was confirmed and the possibility of subdural haematoma seemed strong.

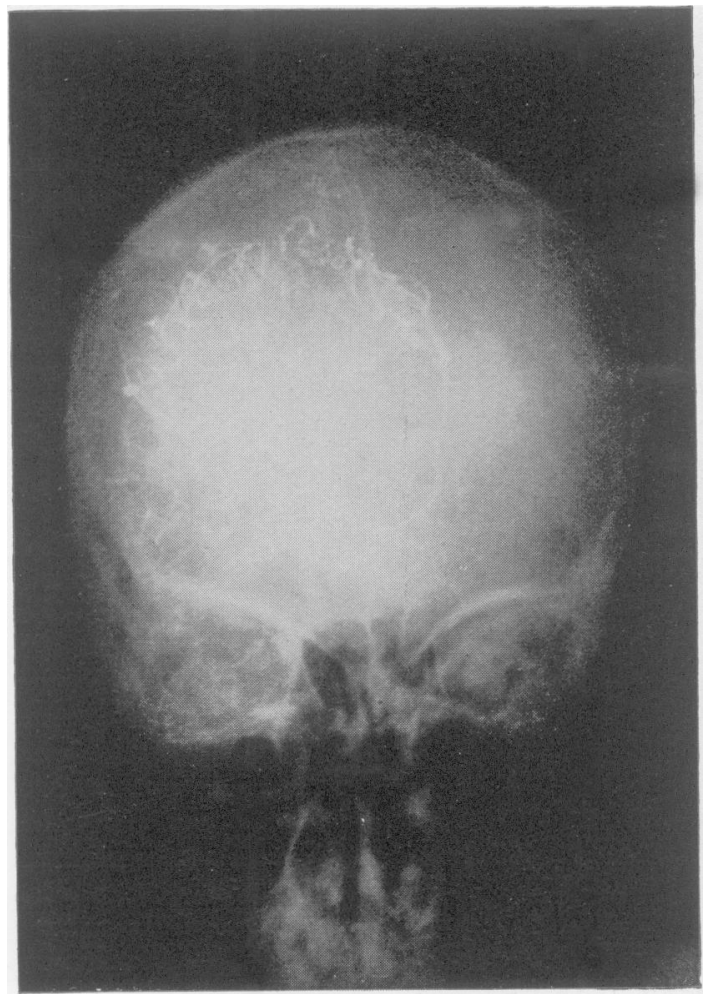

FIG. 5.-Right carotid angiogram. The anterior cerebral artery is shifted to the left and there is an avascular segment over the convexity. This appearance is typical of a subdural collection.

A right carotid angiogram was performed under local anaesthetic and this showed a marked shift of the anterior cerebral artery from right to left and a pattern of vessel displacement suggesting a subdural collection (Fig. 5). She was taken to theatre and a subdural haematoma evacuated on the right side. Despite her age she made an excellent recovery and within three weeks was up and about and sent to the convalescent hospital.

\section{Case No. 4}

A 57-year-old female patient was admitted to the General Infirmary at Leeds as a 'stroke' on December 3, 1958. One month previously she had had an attack of uselessness of the right hand and arm which recovered. The day before admission she had a stroke involving the left hemisphere with dysphasia and flaccid weakness of the right arm and leg. On first examination it seemed that the carotid pulsations were equal, but an hour or two later the left carotid pulsation was clearly less good than the right.

The traditional clinical subdivision of this disorder into cerebral haemorrhage, thrombosis and 


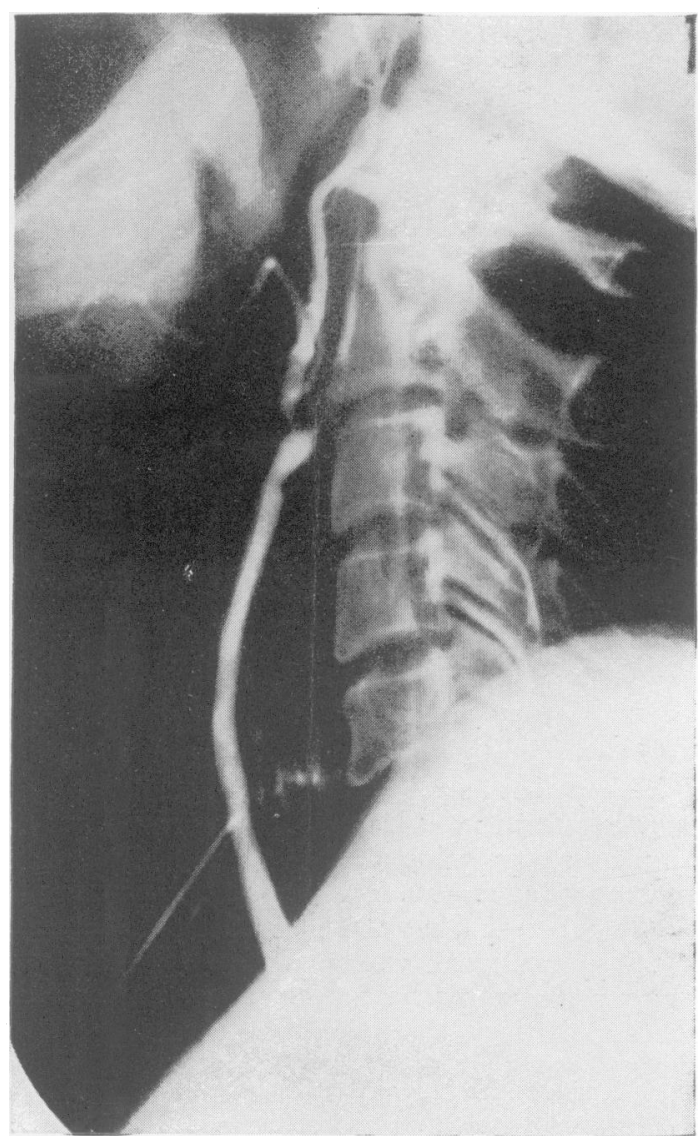

FIG. 6.-Left carotid angiogram. The common carotid artery is irregular and narrowed. The internal carotid artery is practically occluded at its origin, only a little trickle of medium passing beyond. The external carotid artery is partially occluded at its origin.

embolism has been for long based on clinical signs and symptoms of alleged specificity. The common discrepancy between the clinical diagnosis and the post-mortem findings is a fact which challenges the usual diagnostic principles. Current investigations show that a fair proportion of 'strokes' may be due to carotid occlusions or may have intracerebral clots. Angiography may therefore facilitate a more precise diagnosis than previously and point the way to possible surgical treatment.

Left carotid angiography under local anaesthetic was performed and on account of the clinical findings the injection was made low into the common carotid artery. The radiogram showed a partial block at the carotid bifurcation with a trickle of medium passing up the internal carotid artery (Fig. 6).

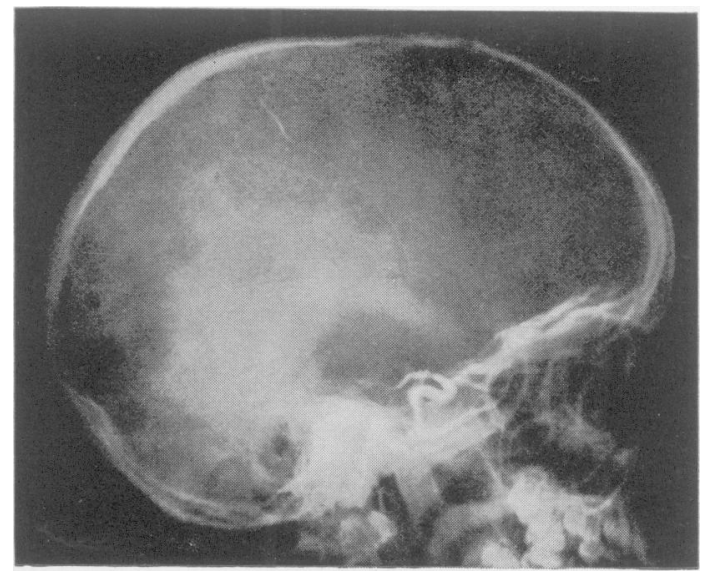

FIG. 7.-Right carotid angiogram. This shows a complete occlusion of the internal carotid artery at its intracranial bifurcation.

The patient was taken to the operating theatre, an atheromatous plaque at the bifurcation of the carotid artery removed and patency of the interral carotid artery restored. Post-operatively she was much more alert and was withdrawing the previously flaccid right limbs away from painful stimuli. The outlook seemed favourable, but she unfortunately developed respiratory difficulty and, despite tracheotomy, died of a diffuse bronchopneumonia.

In the light of subsequent experience and that of others ${ }^{4}$ such a case as this would not be tackled surgically, despite the radiological evidence of a partial carotid occlusion, in view of the density and duration of the neurological signs which suggest extensive hemisphere infarction. Where the signs are minimal or transient, however, and the angiography discloses a partial carotid occlusion, surgery may well yield a gratifying and useful result.

\section{Case No. 5}

A ro-year-old schoolgirl. Two days before admission to hospital she was buying sweets in a shop when she was suddenly taken ill, fell down and was apparently completely unresponsive. When she recovered shortly afterwards she was noticed to have some left-sided weakness but was able to get home without difficulty. Later the same evening the weakness had disappeared and, apart from the child being out of sorts, there was nothing else remarkable. Thirty-six hours later she began to be drowsy and apathetic and was admitted to the General Infirmary at Leeds. Examination revealed a marked left flaccid weakness and a mild left hemiparesis. During the course of a few hours following admission, how- 


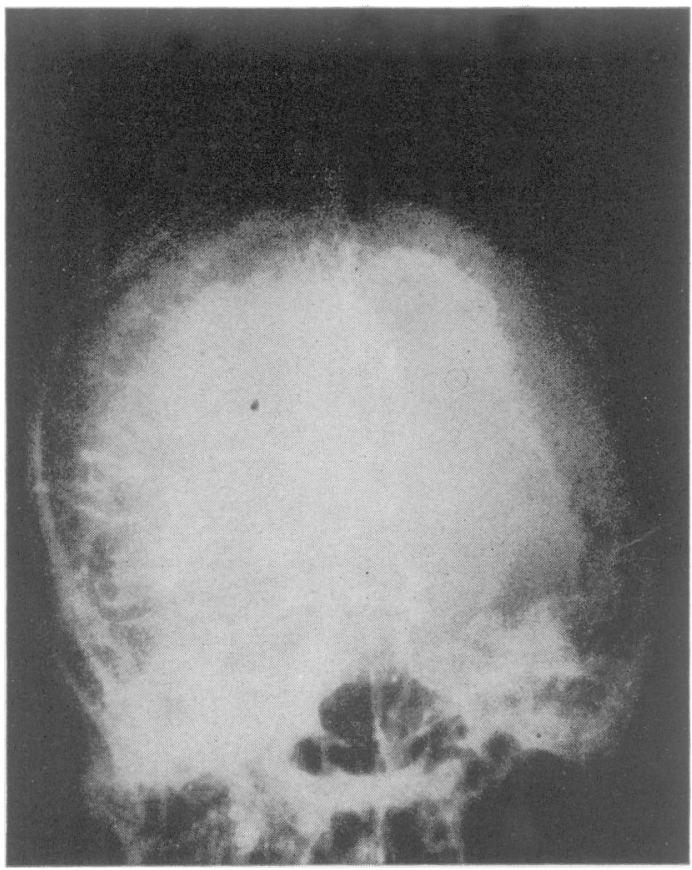

FIG. 8.-Right carotid angiogram. The antero-posterior film shows a shift of the anterior cerebral artery to the left.

ever, she became extremely drowsy and the pulse rate became slower.

This was a difficult diagnostic problem. The dramatic onset, coupled with the initial left-sided hemiparesis, suggested a cerebral accident, a vascular lesion or possibly a fit. There are many causes of listlessness and drowsiness in childhood, but paediatric advice was sought and general causes of the illness eliminated. On account of her deterioration, further investigation was necessary.

Angiography showed a complete block of the right internal carotid artery at the intracranial bifurcation (Fig. 7). She was taken to the operating theatre, and under light general anaesthesia an extensive right lateral craniotomy performed. When the bone flap had been reflected the dura was found to be tight, containing a very full and swollen brain. When the dura was opened the brain looked swollen, pale and ischaemic. An adequate and extensive decompression was performed. The patient made an excellent recovery. She had a mild residual weakness of the left leg but walked well. There was a moderate spastic weakness of the left hand and arm, but she was able to use the hand and arm for everything except fine movements. The child's mental state and ability to learn were unimpaired and she has returned to normal schooling.

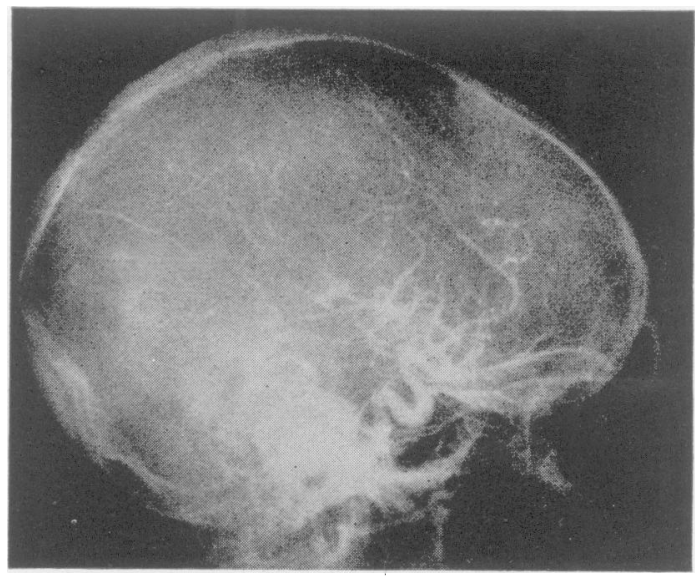

FIG. 9.-Right carotid angiogram. The lateral film demonstrates stretched vessels around an avascular space-occupying lesion in the parietal area.

\section{Ca;e No. 6}

Male patient, aged 37 years. This man enjoyed good health and was leading an active business life until four days prior to admission at the General Infirmary at Leeds on November 22, $195^{8}$. While climbing some steps at work he noticed his left leg was weak and he had to be assisted. Shortly afterwards he felt unwell and nauseated. During the next three or four days he had fluctuating weakness of both left limbs, in addition to headache and occasional sickness. There was no previous history of ear trouble or of chest infection. On examination he was apathetic and listless, there was early papilloedema and a mild left hemiparesis, motor and sensory. Careful examination of the patient revealed no source of infection. He had, however, a temperature around $99^{\circ} \mathrm{F}$. The mild papilloedema, together with headache, listlessness and vomiting suggested a space-occupying lesion, and the lateralizing signs pointed to right hemisphere involvement.

Right carotid angiography was performed under local anaesthesia, an initial film being taken low in the neck to exclude the possibility of carotid occlusion. The films showed an avascular spaceoccupying lesion in the right parietal region, and a shift of the anterior cerebral artery from right to left (Figs. 8 and 9). The patient was transferred to the operating theatre, and an exploratory right parietal burr hole made. Surprisingly, a moderate-sized cerebral abscess was found and evacuated; I c.c. of thorotrast contrast medium was inserted into the cavity to control further treatment (Fig. 10). The abscess was successfully treated by repeated aspiration and by antibiotics. The hemiparesis recovered completely and the patient was discharged home perfectly fit in two 


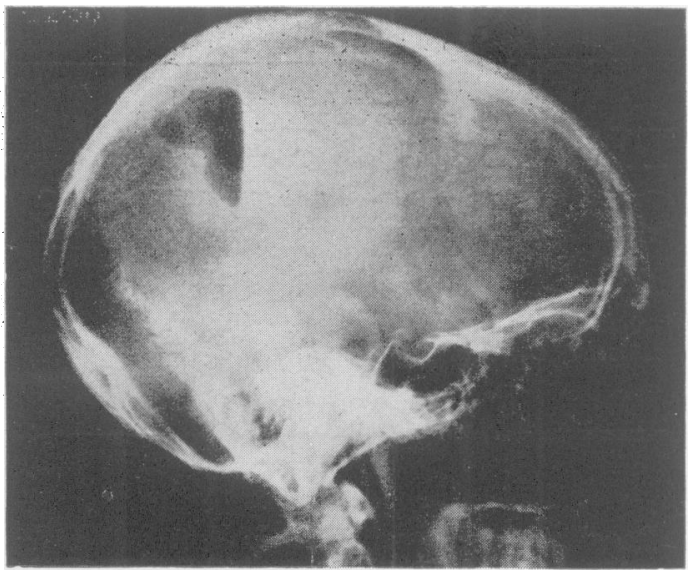

Fig. 10.-A post-operative film taken in the face down position demonstrating the abscess cavity containing thorotrast and air.

and a half months, and he resumed his business activities.

\section{Summary}

The value of cerebral angiography in neurosurgical emergencies is discussed. The authors consider that the procedure is relatively safe and may provide much useful information, given good co-operation between radiologist and neurological surgeon.

The technique and indications are described. The importance of careful clinical assessment of cases before proceeding to angiography is stressed. The method and its value are illustrated in six patients.

\section{Acknowledgments}

We wish to thank Mr. W. R. Henderson, Professor N. M. Dott, Professor A. S. Johnstone and Dr. J. M. Winn for advice during the course of this work and the preparation of this paper.

\section{REFERENCES}

I. BROADRIDGE, A. T., and LESLIE, E. V. (1958), Brit. $\mathscr{f}$ Radiol., 3I, 556.

2. BROWN, A. S. (1955), Anaesthesia, ro, 346.

3. RIISHEDE, J. (1957), Acta psychiat. scand., Suppl. I 18 , Vol. 32.

4. ROB, C., (1959) Proc. roy. Soc. Med., 52, 549.

\section{ENDOCRINE TUMOURS}

(Postgraduate Medical Journal, March 1960)

Price 6s. 6d. post free

ISLET-CELL TUMOURS AND PEPTIC ULCERATION

James B. Gibson, M.D., M.R.C.P.(Edin.), and Richard B. Welbourn, M.A., M.D.(Camb.), F.R.C.S.

ISLET-CELL TUMOUR OF THE PANCREAS, WITH HYPOGLYCAEMIA

Robert S. Monro, F.R.C.S.

PHAEOCHROMOCYTOMA

J. T. Wright, D.M., M.R.C.P.

\section{VIRILIZING SYNDROMES}

Ivor H. Mills, Ph.D., M.D., M.R.C.P.

BILATERAL POLYCYSTIC OVARIES

David Ferriman, D.M., M.R.C.P.

FEMINIZING TUMOURS OF THE TESTIS

P. Paton Philip, M.Chir., F.R.C.S.

CONN'S SYNDROME

T. M. Chalmers, M.D., M.R.C.P.

Published by

THE FELLOWSHIP OF POSTGRADUATE MEDICINE

60, Portland Place, London, W.1 